\title{
Prevalence and distribution of Prototheca species among dairy herds in Poland between 2015 and 2017
}

\section{Zofia Bakuła1, Kukasz Wlazło², Mariola Bochniarz' ${ }^{3}$ Tomasz Piech ${ }^{3}$, Władysław Wawron ${ }^{3}$, Henryk Krukowski², Tomasz Jagielski1}

${ }^{1}$ Department of Applied Microbiology, Institute of Microbiology, Faculty of Biology, University of Warsaw, I. Miecznikowa 1, 02-096 Warsaw, Poland

2Department of Animal and Environmental Hygiene, University of Life Sciences in Lublin, Akademicka 13, 20-950 Lublin, Poland

${ }^{3}$ Department and Clinic of Animal Reproduction, Faculty of Veterinary Medicine, University of Life Sciences in Lublin, Głęboka 30, 20-612 Lublin, Poland

OBJECTIVES: Prototheca spp. are unicellular, colorless, saprophytic, yeast-like algae ubiquitously distributed in the environment, with a particular predilection for moist areas. Occasionally, the algae may cause opportunistic infections in animals and humans. Of the eight currently postulated species, five ( $P$. zopfii, $P$. wickerhamii, $P$. blaschkeae, $P$. cutis, and $P$. miyajifi) are described as the causative agents of protothecosis. $P$. zopfii genotype 2 and $P$. blaschkeae are among the key aetiological agents of bovine mastitis, which persists as the most common and economically important disease of dairy herds worldwide.

The aim of the study was to investigate the prevalence of Prototheca spp. among dairy herds in Poland.

METHODS: The survey included milk samples from 108 dairy cows originating from 23 dairy herds in 13 voivodeships of Poland. Samples with a positive California Mastitis Test (CMT) result (152 samples, 38 cows) were plated on the Prototheca Isolation Medium (PIM). Furthermore, 280 control milk samples (CMT-negative) collected from 70 healthy animals were used for PIM inoculation (Fig. 2.). The plates were incubated at $37^{\circ} \mathrm{C}$ for 72 hours, under aerobic conditions. Each grown isolate was subjected to species identification with both phenotype-based and molecular methods. Conventional differentiation involved micromorphology evaluation and carbohydrate assimilation profiling (API 20C AUX system, Biomerieux ${ }^{\circledR}$, France), while molecular speciation was done using genotype-specific PCR assays for $P$. zopfii genotype $1, P$. zopfii genotype 2 , and $P$. blaschkeae, as described previously [Roesler et al. Int. J. Syst. Evol. Microbiol., 2006, 56:1419-25].

Figure 1. Milk sampling (A); Teat inflammation. (B) California Mastitis Test (C) Watery milk in Prototheca mastitis (D) Prototheca growth on PIM (E)

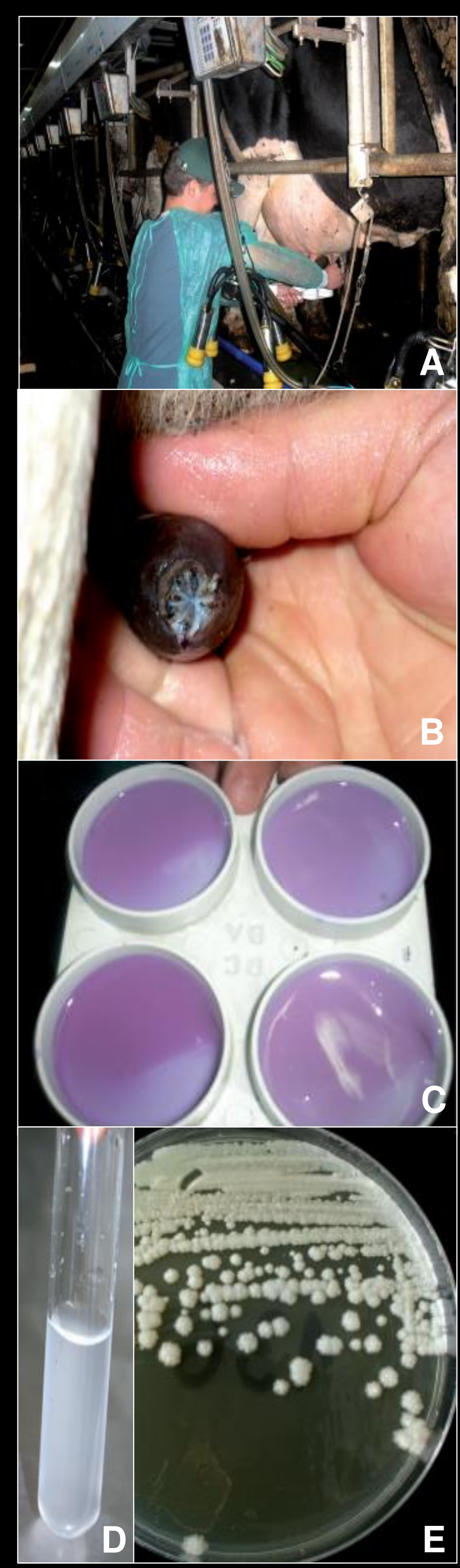

RESULTS: CMT-positive milk samples, collected from $32(32 / 38 ; 84.2 \%)$ cows, yielded 69 (69/152; 45.4\%) Prototheca isolates (Fig. 2.). For 6 (6/38; 15.8\%) cows with mastitis, no Prototheca cultures were obtained. Among isolates cultured, 67 (67/69; $97.1 \%$ ) were identified as $P$. zopfii genotype 2 , and the remaining two were described as $P$. blaschkeae $(2 / 69 ; 2.9 \%)$. All control milk samples (280) were negative for the presence of Prototheca algae.

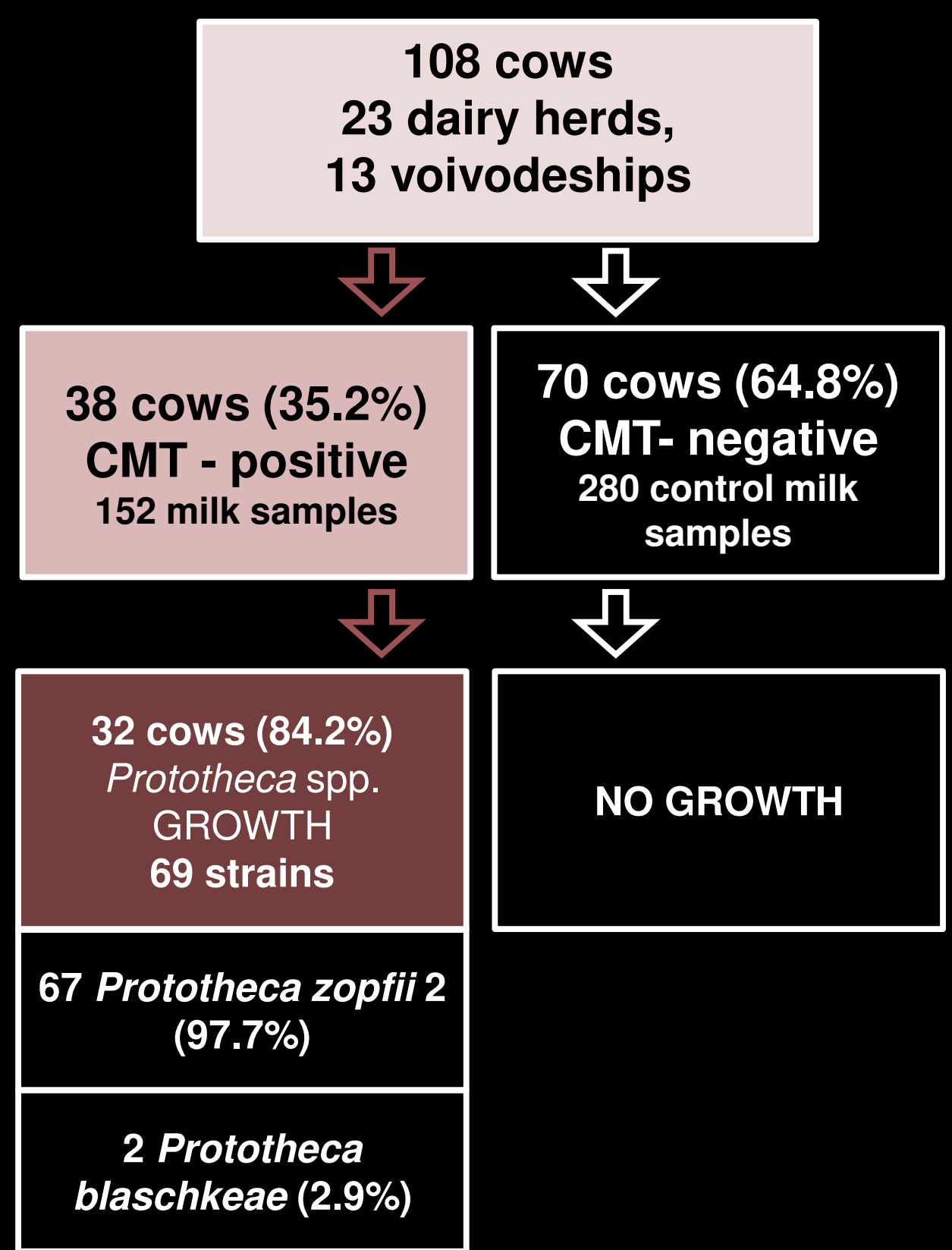

Fig. 2. Flowchart of the study results

CONCLUSIONS: This study clearly showed the predominance of $P$. zopfii genotype 2 as the causative agent of protothecal mastitis among dairy herds in Poland. P. zopfii genotype 2 seems to possess an epidemiological advantage over $P$. blaschkeae in the transmission of the diseases. 